\title{
Capability of TCSC on SSR Mitigation
}

\author{
Rui Zheng, Gen Li, Jun Liang \\ School of Engineering, Cardiff University, Cardiff, UK \\ Email: Zhengr4@cardiff.ac.uk
}

Received December 2014

\begin{abstract}
TCSC (Thyristor-Controlled Series Compensator) has been used in modern power systems to increase power transfer and system stability. Besides, due to its controllable feature, TCSC has been used to work as a solution to sub-synchronous resonance (SSR) problems. However, it is not clear so far whether TCSC's inherent characteristic can avoid SSR and its effectiveness for SSR damping. In this paper, an equivalent circuit of TCSC is used to better understand how TCSC performs at sub-synchronous frequency ranges. Therefore, interactions of TCSC behaviors and SSR components can be viewed individually. The effects of TCSC operation interfered by sub-synchronous components are discussed. Results show that TCSC has the capability to damp SSR, but this capability can be deteriorated if TCSC firing logic cannot be effectively maintained.
\end{abstract}

\section{Keywords}

TCSC, SSR, Frequency Response

\section{Introduction}

TCSC (Thyristor Controller Series Compensator) is one of the FACTS (Flexible AC Transmission System) devices that have been proved to be practical in modern power systems. The benefits of TCSC devices are: increasing power capability; improving power stability and mitigating SSR (sub-synchronous resonance) phenomena. The first TCSC project was commissioned in 1992, the Kayenta substation, in the USA [1]. Other typical TCSC projects include the Stode project [2] and Yimin-Fengtun TCSC project [3]. Recently, the UK National Grid has decided to install a TCSC device to increase the stability of Scottish-England transmission system as a large number of wind power generations are located in Scotland [4]. This draws attention from both industry and academic researchers again.

SSR is a drawback of series compensators. The reason for SSR occurrence is that electrical frequency co-ordinates with the nature frequency of mechanical shafts system [5]. When SSR happens, it can cause damages to turbine shafts and must be avoided. Since it is impossible to determine the mechanical shaft masses, the solutions to SSR problems are mostly located in the electrical systems.

Conventional solutions to SSR are using filters or by-pass circuit breakers. The filters are installed at the generator side or compensator side, and block certain frequency which is recognized through analysis into the system. This method does not suit a fast-varying system as it requires numerous calculations when new generation is inserted. The by-pass circuit breakers are disable series compensators at the cost of system stability problems when SSR is observed. 
TCSC, as a controllable device, enables application of low-level control strategy to regulate TCSC characteristic to damp SSR problems. The $220 \mathrm{kV}$ Kayenta substation [1] uses TCSC equipped with closed-loop current controller to help solve SSR problems. In the Stode project [2], SVR (Synchronous Voltage Reversal) scheme is applied to maintain TCSC apparent reactance hence SSR problem is solved. It is also shown that TCSC behaved inductive in most sub-synchronous frequency ranges thus SSR cannot occur. However, as TCSC characteristic is related to its firing scheme, it cannot be distinguished whether it is TCSC's inherent characteristic or extra controller that mitigates SSR.

Some efforts have been done to investigate TCSC characteristics. Paper [6] researched on TCSC's damping against SSR. By using a complex torque coefficient approach, system electrical damping characteristics within sub-synchronous frequency ranges based on different combinations of TCSC and FSC are obtained. It was indicated that TCSC cannot bring in additional damping but it moved damping at some frequencies to other frequencies. Therefore, in some cases, TCSC could offer better damping than FSC but in some cases it becomes a worse option. This idea was proposed but not enough explanation is given.

This paper further analyzed this idea in [6] and aims to figure out the interaction between the dynamic behaviors of TCSC and SSR phenomena. A method to predict TCSC's damping SSR performance is given by testing whether an equivalent circuit is performing inductive or capacitive at concerned frequency. The occurrence of inductive or capacitive behavior is derived during the interaction of TCSC dynamic and SSR, and is used to explain why TCSC can worsen or improve SSR damping performance.

\section{Principle of TCSC Operation}

\subsection{Triggering of TCSC}

TCSC consists of a TCR (Thyristor-controlled Reactor) in parallel with a fixed capacitor. By changing the instants that thyristors are fired, the current flowing through TCR will vary thus the apparent reactance of TCR and TCSC device could be controlled. The typical single-line diagram of TCSC is shown in Figure 1(a). Also, since the TCR reactance is fixed in steady state, the TCR is approximated to an equivalent inductor as shown in Figure 1(b).

The firing instants are defined using a variable, firing angle $\alpha$. This $\alpha$ can be transformed into electrical degree from fundamental cycle time. By definition, the start point of a firing angle is determined by the voltage zero-crossing points. However, in practical TCSC projects, as capacitor voltage is less stable than line current, therefore line current is preferable as a reliable input to determine the firing instants. Since TCSC behaves capacitive in normal operation, the line current $(I)$ leads $90^{\circ}$ ahead of capacitor voltage $\left(V_{C}\right)$. Thus, a newly formed firing angle is defined as

$$
\alpha_{i}=\alpha+90^{\circ}
$$

where $\alpha_{i}$ denotes the new firing angle starts from current zero-crossing points. Only capacitive conditions are considered throughout this paper.

When a TCSC device is fired under steady state conditions, the typical waveforms of its inductor current $\left(I_{L}\right)$, capacitor voltage $\left(V_{C}\right)$ and line current $(I)$ are shown in Figure 2.

The synchronization between current $I$ and thyristor triggering pulses is shown in Figure 2. A practical approach is to detect the zero-crossing points of current $I$. Once a zero-point is detected, this instant is recorded as a reference, e.g. $t_{0}$. The desired firing angle $\alpha_{i}$ is transformed into time delay and then applied onto the instant $t_{0}$. After certain time delay, a pulse is generated and will be sent to thyristor to trigger the current conduction. The triggering pulses are in sign with capacitor voltage at that instant. In Figure 2, pulse "a” is generated

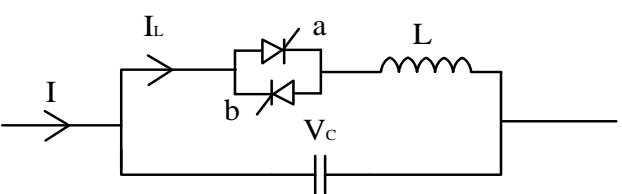

(a)

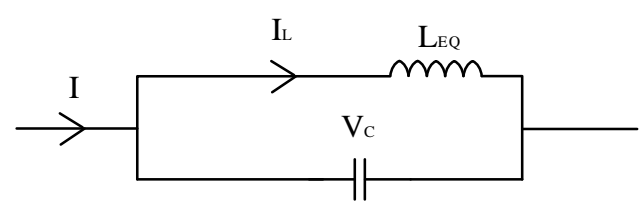

(b)

Figure 1. Schematic diagrams of TCSC and its equivalent circuit. (a) Schematic diagram of TCSC; (b) Equivalent circuit of TCSC. 
when capacitor voltage is positive, thus it is used to trigger thyristor “a”. In this waythyristors are fired with accordance to line current and selected firing angles. This procedure is repeated to maintain TCSC triggering synchronization.

\subsection{TCSC Dynamics Considering Impacts of SSR}

TCSC performance depends on thyristor firing scheme, the interference of sub-synchronous components in capacitor voltage can cause TCSC behavior varied if the firing scheme is not regulated with accordance to subsynchronous components. Figure 3 shows a simple example of the interruption of sub-synchronous voltages when TCSC is fired with constant firing angle.

In reality, induced sub-synchronous components are quite small (only around 1\% in magnitude compared with fundamental component), therefore, most models in previous studies assume that TCSC on/off switching is not affected. However, this initial small difference can be amplified if no external controllers are installed, and the synchronization of TCSC triggering is lost. In Figure 3, with the penetration of sub-synchronous capacitor voltage component, the frequency of newly-formed voltage is slightly changed, thus the half-cycle time between two zero-crossing points is different. If the firing logic takes constant delay (firing angle) from the last current zero-crossing point, the thyristor conducting time is varied.

The first half-cycle of the newly-formed capacitor voltage $V_{C}$ (solid line), as shown in Figure 3(a), is increased due to penetration of sub-synchronous components. This results in longer thyristor conducting interval, hence the inductor current is increased compared with that in Figure 2. On the contrary, in Figure 3(b), the first cycle of capacitor voltage $V_{C}$ is reduced, thus the inductor current $I_{L}$. Note that the sub-synchronous voltage that induced in the circuit is quite small in magnitude compared with fundamental component, i.e. around only $1 \%$. Thus the effective value of capacitor voltage is almost constant. But the inductor current is more sensitive to these changes. Considering the half cycle in Figure 3, the apparent reactance of TCR $\left(X_{\text {TCR }}\right)$ is decreased due to the increasing $I_{L}$ and decreasing $V_{C}$. In the second half cycle, the thyristor conducting time is shorten hence corresponding $I_{L}$ is decreasing. This results in larger $X_{\mathrm{TCR}}$. As TCSC is actually a parallel circuit of TCR and fixed capacitor, if $X_{\text {TCR }}$ varies, the TCSC apparent reactance $\left(X_{\text {TCSC }}\right)$ is changed as well. This

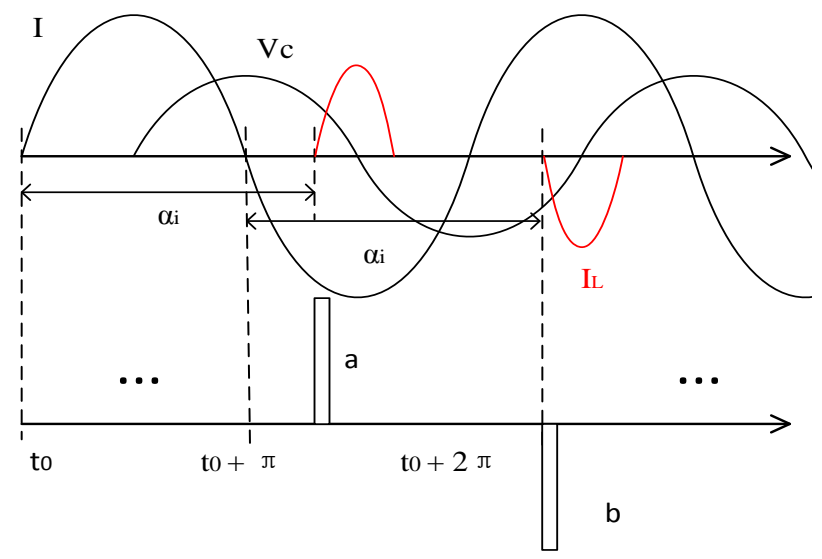

Figure 2. Typical waveforms of inductor current $\left(I_{L}\right)$, capacitor voltage $\left(V_{C}\right)$ and line current $(I)$.

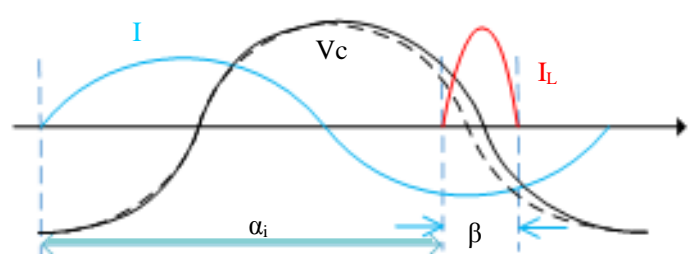

(a)

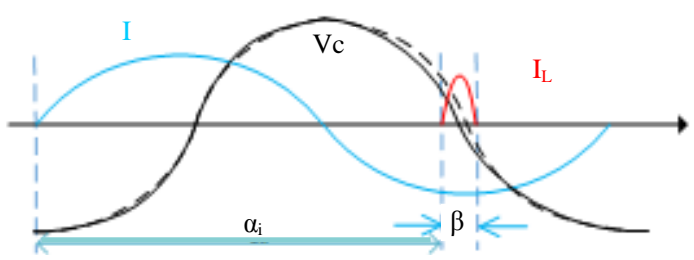

(b)

Figure 3. Waveforms of inductor current $\left(I_{L}\right)$, capacitor voltage $\left(V_{C}\right)$ and line current $(I)$ with sub-synchronous voltage components: (a) First half cycle voltage increased; (b) first half cycle decreased. 
phenomena repeats until the sub-synchronous components are fully damped, thus $X_{\text {TCSC }}$ is observed to be oscillating.

\subsection{The Equivalent Circuit of TCSC}

As the TCSC is operated steadily, $V_{C}, I$ and $I_{L}$ are all constant. Considering TCSC performance within each half cycle, $X_{\mathrm{TCR}}$ can be derived using the effective value of $I_{L}$ and $V_{C}$. As $I_{L}$ contains only fundamental component and its odd order harmonics, Fourier Transform can be used to obtain magnitudes of each component, thus $I_{L}$ can be written as:

$$
I_{L}=I_{k} \cos \left(\omega_{k} t+\varphi_{k}\right)
$$

where $I_{k}$ denotes the magnitude of $k^{\text {th }}$ order harmonic and $k$ is odd integer. Then the effective value of $I_{L}$, denoted by $I_{L \text {,eff }}$, is given by:

$$
I_{L, \mathrm{eff}}=\sqrt{I_{1}^{2}+I_{3}^{2}+\cdots+I_{k}^{2}}
$$

Thus the equivalent inductance of TCR, denoted by $L_{\mathrm{EQ}}$ is given as:

$$
L_{\mathrm{EQ}}=\frac{\sqrt{V_{c, \text { fund }}^{2}+V_{c, \text { sub }}^{2}}}{\omega_{0} \times\left|I_{L, \text { eff }}\right|} \approx \frac{\left|V_{c, \text { fund }}\right|}{\left|I_{L, \text { eff }}\right|}
$$

whereas $\left|V_{c, \text { sub }}\right|$ is the sub-synchronous voltage that is around only $1 \%$ of $\left|V_{c, \text { fund }}\right|$, thus can be ignored.

\section{Characteristic of the Equivalent Circuit}

As the approximation takes fixed inductor and capacitor as its components, the non-linear characteristic caused by TCSC switching can be ignored. Therefore, the frequency response of the paralleled circuit is developed as follow:

$$
Z(\omega)=\frac{X_{C} * X_{L, \mathrm{EQ}}}{X_{C}+X_{L, \mathrm{EQ}}}=\frac{\frac{1}{j \omega C} * j \omega L_{\mathrm{EQ}}}{j \omega L_{\mathrm{EQ}}+\frac{1}{j \omega C}}=\frac{j \omega L_{\mathrm{EQ}}}{1-\omega^{2} L_{\mathrm{EQ}} C}
$$

Assign $Z(\omega)=\frac{j \omega L_{\mathrm{EQ}}}{1-\omega^{2} L_{\mathrm{EQ}} C}>0$, thus the boundary can be derived as

$$
L_{\mathrm{EQ}}<\frac{1}{\omega^{2} C}
$$

Equation (6) indicated that it is possible to derive the inductive behavior range of the equivalent circuit. Actually, as for a TCSC device, the capacitance is fixed. The TCR apparent reactance varies with different operating points, which makes the whole TCSC device to perform differently at concerned sub-synchronous frequencies.

An example is considered here. The compensator is selected to provide $57 \%$ compensation level in FBM system. As TCSC can be tuned to offer around $2 \sim 3$ times of the base capacitance, thus the fix capacitor is selected equal to $20 \%$ of transmission line reactance, which is $34.01 \mu \mathrm{F}$. In this case, from the study of [7], the system suffers torsional mode 1 which is at $15.7 \mathrm{~Hz}$. In order to check the SSR response of the system with different sub-synchronous behaviours, three cases are built to make a critical scenario so that the impacts of inductive or capacitive performance can be studied. The parameters of the three cases are shown in Table 1. The frequency responses for these three cases are plotted using (5) in Figure 4.

Figure 4 illustrates that the resonant point that divides inductive region and capacitive region is moved when the parameter of inductor changes. As $L_{\mathrm{EQ}}$ is derived from instantaneous capacitor voltage and TCR current in TCSC device, when $X_{\mathrm{TCSC}}$ begins to oscillate, $L_{\mathrm{EQ}}$ starts to oscillate as well. When $Z(60 \mathrm{~Hz})$, rises from $-88 \mathrm{ohm}$ to $-94 \mathrm{ohm}$ capacitive, the frequency behavior at $16 \mathrm{~Hz}$ is moved from inductive to capacitive region, denoted by red and black curves in Figure 4. In this way, the circuit cannot remove SSR. On the other hand, if 
$Z(60 \mathrm{~Hz})$ decreases, i.e. from $-88 \mathrm{ohm}$ to $-83 \mathrm{ohm}$ capacitive, the behavior at $16 \mathrm{~Hz}$ is still inductive and corresponding sub-synchronous component can be damped. As the equivalent circuit is derived from impedance response of TCSC device, the theory applies for TCSC as well.

\section{Simulations and Results}

\subsection{Parameters}

The example case considers FBM system with a compensation level of 57\%. The compensator is provided by TCSC or its equivalent circuit only. Table 2 gives the parameters of TCSC and the equivalent circuit parameters.

\subsection{Simulation Results and Analysis}

Figure 5 gives the LPA-LPB torque in the FBM system operated under 57\% compensation level. It is shown that the equivalent circuit of TCSC shows good damping performance against SSR phenomena. In Figure 5, the torque oscillates at around 0.5 p.u. when fault is applied at 4 sec and converges with time past. This indicates that the equivalent circuit can damp the $16 \mathrm{~Hz}$ component due to its inductive behavior at that frequency which is expected using the result shown in Figure 4.

Install TCSC device and remove the equivalent circuit, tuning the firing angle to maintain the same compensation level, the simulation result is shown in Figure 6.

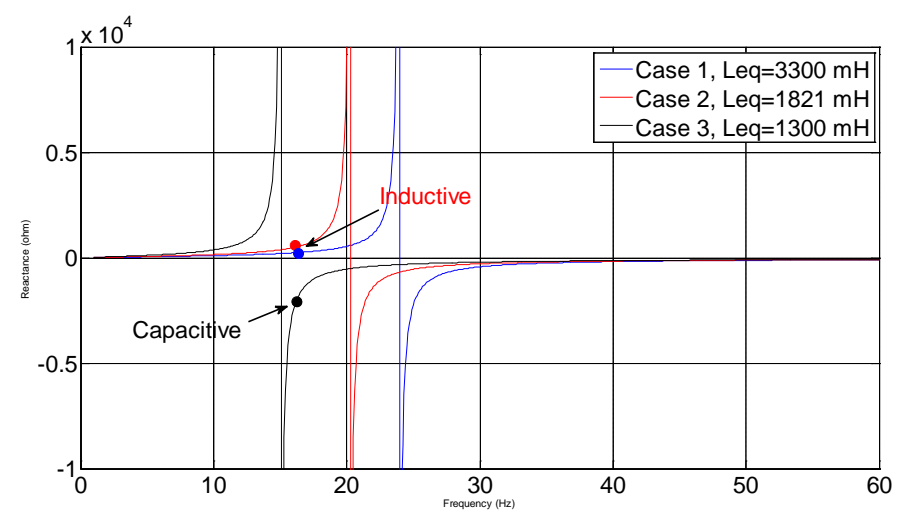

Figure 4. Frequency response of three cases.

Table 1. Three combinations of parameter.

\begin{tabular}{ccccc}
\hline & \multicolumn{2}{c}{ The equivalent circuit } & \multicolumn{2}{c}{ TCSC } \\
\hline Case 1 & $L_{\mathrm{EQ}}=3000 \mathrm{mH}$ & $Z(60 \mathrm{~Hz})=-83 \Omega$ & $\alpha_{i}=256$ & $\alpha=166$ \\
Case 2 & $L_{\mathrm{EQ}}=1821 \mathrm{mH}$ & $Z(60 \mathrm{~Hz})=-88 \Omega$ & $\alpha_{i}=253$ & $\alpha=163$ \\
Case 3 & $L_{\mathrm{EQ}}=1300 \mathrm{mH}$ & $Z(60 \mathrm{~Hz})=-94 \Omega$ & $\alpha_{i}=251$ & $\alpha=161$ \\
\hline
\end{tabular}

Table 2. TCSC and its equivalent circuit parameters.

\begin{tabular}{ccc}
\hline & TCSC & The Equivalent circuit of TCSC \\
\hline Inductance & $23 \mathrm{mH}$ & $1821 \mathrm{mH}$ \\
Capacitance & $34.01 \mu \mathrm{F}$ & $34.01 \mu \mathrm{F}$ \\
Firing angle & $163.5^{\circ}$ & N/A \\
TCSC apparent reactance at $60 \mathrm{~Hz}$ & $-88 \Omega$ & $-88 \Omega$ \\
Compensation level & $57 \%$ & $57 \%$ \\
\hline
\end{tabular}




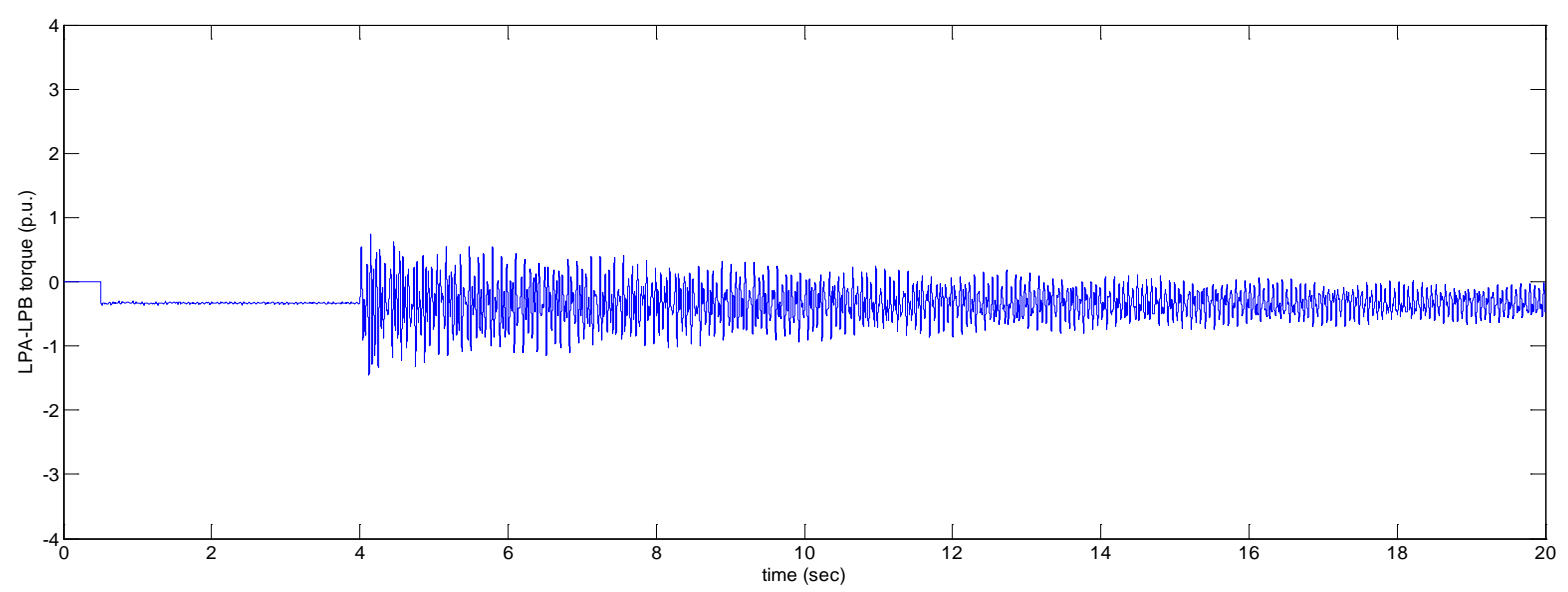

Figure 5. LPA-LPB torque withequivalent circuit of TCSC.

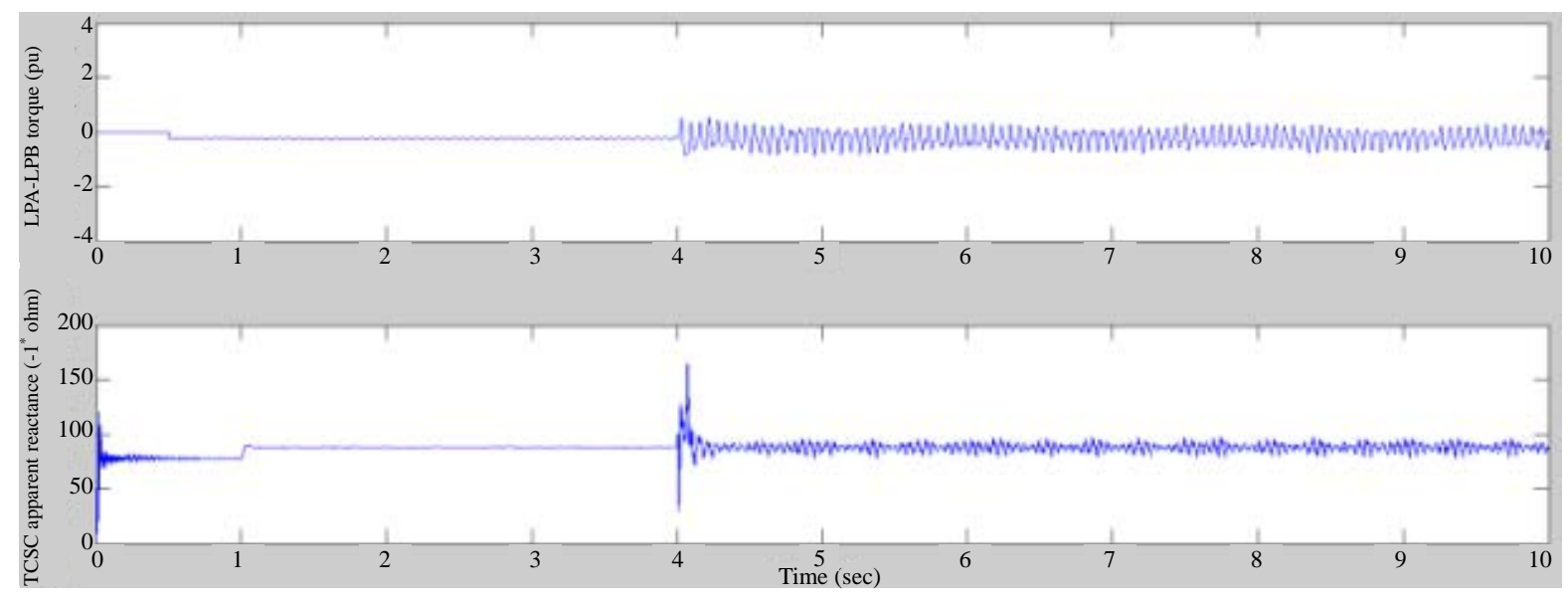

Figure 6. LPA-LPB torque (upper) and TCSC apparent reactance with closed-loop triggering logic (lower).

In Figure 6, the torque gets oscillated at first around 0.5 p.u. and starts to damp after fault clearance. The TCSC reactance plot shows that the impedance controller is maintaining at reference level while a small oscillation due to PI controller can be observed.

The above results show that TCSC can damp SSR problems when its corresponding sub-synchronous behavior is inductive and its characteristic is maintained. This is theoretically derived using the frequency response of the equivalent circuit. Therefore, it is necessary to validate whether a capacitive behavior would amplify SSR problems.

\section{Validation and Results}

When TCSC reactance starts to oscillate using open-loop scheme, the possible reactance oscillation range can be various. As shown in Figure 4, under some conditions, the behavior at $16 \mathrm{~Hz}$ may turn into capacitive. Consider case 3 in which the equivalent reactance is selected to be $-83 \mathrm{ohm}$, as shown in Table 3 .

As shown in Figure 4, the circuit behaves capacitive at $16 \mathrm{~Hz}$, hence the circuit is expected to amplify SSR problem. The simulation result is shown in Figure 7.

Figure 7 shows that the LPA-LPB torque gets oscillated as fault is applied. This oscillation increases very fast to 15 p.u. in 2 seconds. This indicates that capacitive behavior cannot solve SSR problems but amplify the sub-synchronous components to result in further oscillation.

Equip TCSC into system to replace the equivalent L-C circuit, and change firing angle to make TCSC fundamental reactance equal to $-83 \mathrm{ohm}$. From the previous analysis, the sub-synchronous behavior at $16 \mathrm{~Hz}$ is capacitive in this case. Simulation result is shown in Figure 8. 
Table 3. Parameters of the equivalent circuit of TCSC.

\begin{tabular}{cc}
\hline Inductance & $3000 \mathrm{mH}$ \\
\hline Capacitance & $34.01 \mu \mathrm{F}$ \\
TCSC apparent reactance at $60 \mathrm{~Hz}$ & $83 \Omega$ \\
Compensation level & $51 \%$ \\
\hline
\end{tabular}

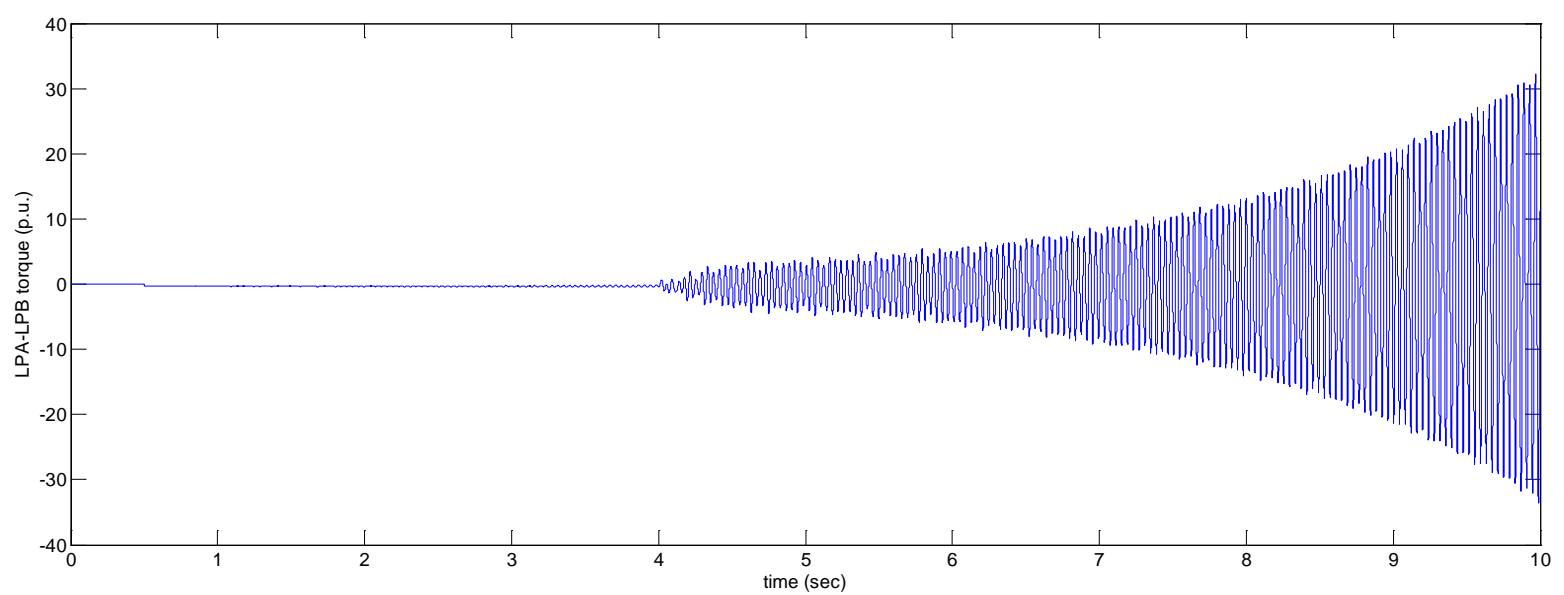

Figure 7. LPA-LPB torque withequivalent circuit of TCSC.
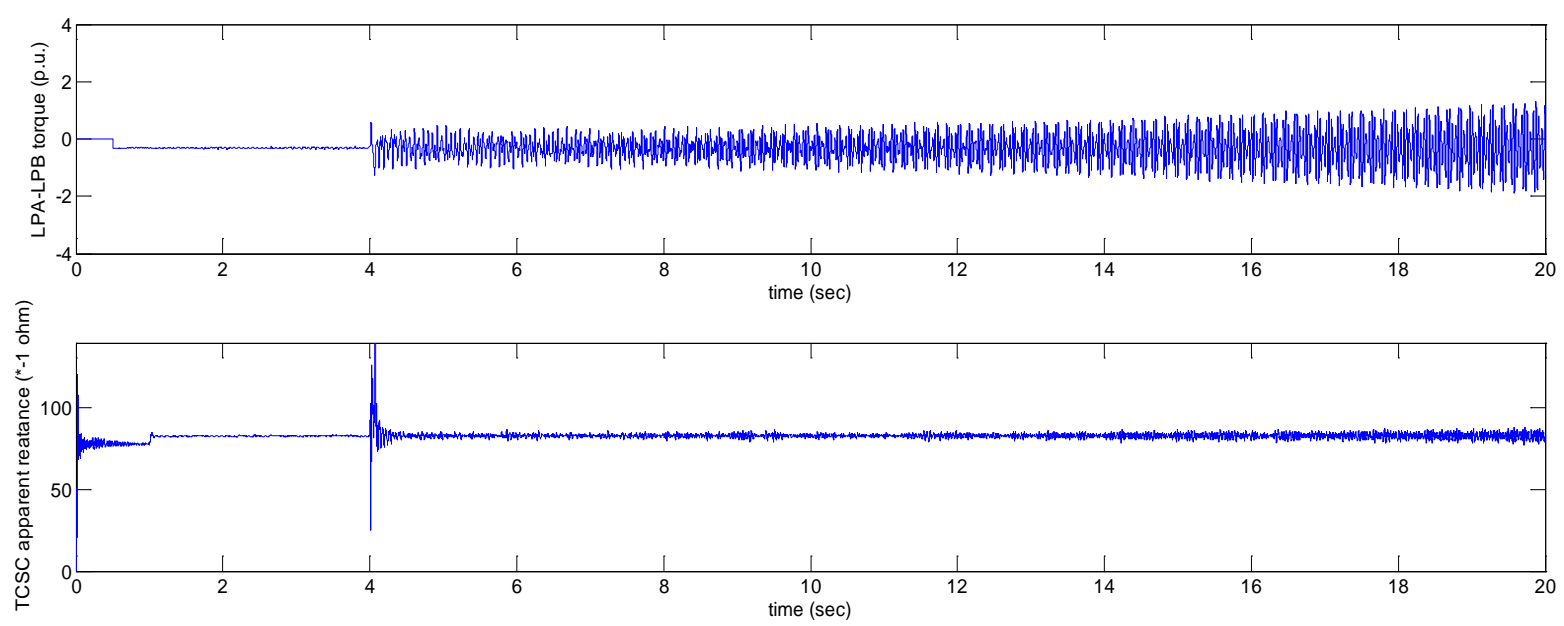

Figure 8. LPA-LPB torque (upper) and apparent reactance response of TCSC with closed-loop controller (lower).

Figure 8 shows that even TCSC device can not solve SSR problems under this operating condition. However, compared with the equivalent circuit in Figure 7, the torque oscillation amplitude remains much lower, which is 0.5 p.u. at 4 sec to 2 p.u. at 20 sec. This difference between TCSC and equivalent circuits performance indicate that similar sub-synchronous behaviors, either inductive or capacitive. However, the active regulating characteristic of TCSC has better impacts than the equivalent circuit in damping SSR problems.

The results validate the relationships between inductive or capacitve sub-synchronous behaviors and corresponding SSR damping performances. It is indicated that SSR can be solved if a circuit provides inductive behavior at concerned frequency, while capacitive behavior will amplify SSR problems.

\section{Conclusion}

The frequency response of the equivalent circuit is obtained and it illustrates that the circuit can damp SSR at 
concerned sub-synchronous frequency if corresponding behavior is inductive. This sub-synchronous behavior will be moved if the equivalent inductance is varied, which is related to dynamic of TCR. TCSC impedance stability is related to its SSR damping performance, as long as its sub-synchronous behaviors at concerned frequency are inductive. However, it is also noted that the dynamic of TCSC may sometimes turn its behavior into capacitive, which will lead to a SSR failure. Therefore, a proper controller to limit the output of TCSC apparent reactance or other variables is helpful to improve SSR performance. The capacitive sub-synchronous behavior has been validated to be amplifying SSR problems, but the comparison between TCSC and its equivalent circuit shows that even in the same capacitive conditions, TCSC's inherent regulating has better impacts on decreasing the slope of oscillations.

\section{Acknowledgements}

The author gratefully acknowledges Jun Liang for his advice and guidance throughout this research, and Gen Li for preparing most of the figures and plots. Without their help, it is impossible to complete this paper.

\section{References}

[1] Christi, N., Hedin, R., Johnson, R., Krause, P. and Montoya, A. (1991) Power System Studies and Modelling for the Kayenta 230 kV Substation Advanced Series Compensation. International Conference on AC and DC Power Transmission, 17-20 September 1991, 33-37.

[2] Holmberg, D., Danielsson, M., Halvarsson, P., et al. (1996) The Stodethyristor Controlled Series Capacitor. CIGRE, Paris, 14-105.

[3] Liang, J., Guo, J.B. and Zhou, X.X. (1998) Theory Analysis and Engineering Study of Yimin-Fengtun 500 kV TCSC Transmission System. International Conference on Power System Technology, 1, 377-381.

[4] ENSG. Our Electricity Transmission Network: A Vision for 2020. https://www.gov.uk/government/uploads/system/uploads/attachment_data/file/48274/4263-ensgFull.pdf

[5] Subsynchronous Resonance Working Group (1980) Proposed Terms and Definitions for Subsynchronous Oscillations. IEEE Transactions on Power Apparatus and Systems, PAS-99.

[6] Wu, X. and Jiang, P. (2010) Borrow Damping Phenomena of TCSC to Sub-Synchronous Resonance. Power System Protection and Control, 38.

[7] IEEE Subsynchronous Resonance Task Force of the Dynamic System Performance Working Group Power System Engineering Committee (1977) First Benchmark Model For Computer Simulation of Subsynchronous Resonance. IEEE Transactions on Power Apparatus and Systems, PAS-96. 